\title{
Laser Beam Shaping and Mode Conversion in Optical Fibers
}

\author{
Mona MAYEH ${ }^{1}$ and Faramarz FARAHI ${ }^{1,2}$ \\ ${ }^{1}$ Center for Optoelectronics and Optical Communications, University of North Carolina at Charlotte, 9201 University \\ City Boulevard, Charlotte, NC, 28223, USA \\ ${ }^{2}$ Department of Physics and Optical Science, University of North Carolina at Charlotte, 9201 University City Boulevard, \\ Charlotte, NC, 28223, USA \\ *Corresponding author: Faramarz FARAHIＥ-mail: ffarahi@uncc.edu
}

\begin{abstract}
A new class of all-fiber beam shaping devices has been realized by inverse etching the end face of single mode and multimode fibers to form a concave cone tip. Concave tip fiber can convert a Gaussian beam profile to a flat top beam profile with a uniform intensity distribution. A flat top beam with intensity variation of approx. 5\% and flat top diameter to spot diameter ratio of $67 \%$ has been achieved. This device can also change the beam shape from a Gaussian to a donut by moving the observation plane. A flat top multimode fiber beam delivery is attractive for applications which require high power and uniform intensity distribution. In single mode fiber, concave tips could be used to reduce the beam spot size diameter, enabling efficient light coupling from a single mode fiber to an integrated optical waveguide.
\end{abstract}

Keywords: Fiber beam shaping, fiber mode conversion, laser beam shaping

\section{Introduction}

Laser beam shaping has been a subject of research over the years for many practical reasons. On one hand a uniform intensity (flat top) profile has found applications in laser writing, material processing, industrial machining, photolithography, medicine, and on the other hand mode conversion for effective coupling of light into small size waveguides is very important in optical communication and sensors. Laser beam shaping attempts go back to four decades ago, when Frieden et al. introduced conversion of a Gaussian profile into a plane wave of uniform irradiance [1]. Since then several techniques have been proposed to create a flat top and collimated beam with homogenous irradiance, from a Gaussian beam mainly radiating from laser [2]. These methods include filters with radially varying absorption profiles [3], diffractive elements like holograms [4-6] and refractive optics $[7,8]$ which convert a Gaussian to a flat top beam. Although capable of transferring Gaussian to flat top, these methods have their own limitations: modest efficiency, low fabrication tolerance, alignment control and wavelength sensitivity, to name a few. The more recent attempts toward achieving a flat top beam include fiber based optical beam shaping owing to its low attenuation loss, compact design and flexibility in delivering the laser light. Square core jacketed air-clad fiber [9] has been proposed to deliver flat top, high power beams using multimode fibers. However, the cost of this kind of specialty fiber is much higher than most standard fibers. There have been few reports on fiber beam shaping using all-fiber long period grating (LPG) [10-12] and single mode abrupt tapered fibers [13]. The loss

Received: 28 September 2010 / Revised version: 28 October 2010

(C) The Author(s) 2011.This article is published with open access at Springerlink.com 
and wavelength dependency of LPG limit the applications of this method.

Despite many research and development efforts in the area of integrated optics, efficient fiber-to-waveguide coupling remained a challenge particularly for applications in optical communication and integrated optical sensors. The coupling loss in the fiber-waveguide interface arises from the mode mismatch between the fiber and optical waveguide and index difference (Fresnel loss). To resolve the low coupling efficiency problem, several methods have been proposed, which require various mode conversions either on fiber side or in the integrated waveguide region. Except for a few reported works related to fiber mode converters [14, 15], most attempts for improving the coupling efficiency between fiber and waveguide have been focused on waveguide design and geometry. These coupling methods can be mainly categorized into: 1) vertical coupling such as grating assisted coupling and 2) transverse coupling which involves various spot size conversions. E. Di Fabrizio et al. demonstrated an efficient fiber-to-waveguide coupling method by fabricating a diffractive micro-lens element directly on tip of the fiber $[14,15]$. In this method a $10-\mu$ m-diameter Gaussian beam from the fiber was reshaped and focused into a 5.2- $\mu \mathrm{m}$-diameter beam. Despite many advantage of this method such as ease of alignment, self-focusing property and control over beam size, the fabrication process is complicated. This method showed an improvement in coupling efficiency from fiber to a waveguide, but 5-micron mode field diameter is still very large in comparison with mode filed diameters of many integrated optical waveguides. Several configurations have been proposed for improving fiber-to-waveguide coupling efficiency in very small size waveguides such as grating coupler [16-18], micro-ring vertical coupler [19], parabolic reflector [20], and tapered waveguides [21-25].

In this paper we describe a method to change the intensity profile of an optical fiber in a controlled way. We apply this method to generate flat and annular output beam profiles for single mode and multimode fibers, as well as a mode converter for efficient coupling from a single mode optical fiber to an integrated optical waveguide. This method is based on shaping the fiber end tips as will be described in the following section. We then present theoretical and experimental results.

\section{Concave tip fiber design and fabrication}

Chemical etching of silica fiber has been developed due to its wide application in near-field optical scanning microscopy [26], fluorescence sensors [27], and thermal probe sensors [28]. In comparison to other etching methods, wet chemical etching offers a simple and inexpensive fabrication technique [29]. Chemical etching of fiber occurs due to the reaction between hydrofluoric (HF) acid and fused silica immersed in HF solution. It has been shown that the addition of a germanium dopant to fused silica changes the etching rate of dopant/silica relative to pure silica [30].

The surface profile of fiber end face can be controlled by several parameters such as etching time, acid to water ratio and temperature of working environment. There have been reports of engineering fiber end profile in the concave form and convex form for fiber alignment applications [31] and fiber Fabry-Perot strain sensors [32].

For our experiments we chose single mode fiber, SMF-28, and Infinicore 300 multimode fibers, which both having germanium doped core. About 1 $\mathrm{cm}$ of the jacket from the end of each fiber was removed, and fibers were cleaved. A setup described by Demagh et al. [31] was used to etch the fibers' tips (Fig. 1). Etching process was carried out in 49\% HF. Temperature was kept constant at $25{ }^{\circ} \mathrm{C}$ during the experiment. After etching, the fibers were removed and rinsed with deionized water and left to dry. As the reaction between $\mathrm{HF}$ and the core material initiated, the fiber end changed from the initial cylindrical form to a conical form (Fig. 2). Initially, etching of fiber core was dominant giving 
rise to the formation of a concave cone (Fig. 2(b)) while the top of this cone was centered to the axis of the fiber core. As the diameter of the fiber decreased due to chemical attack to the clad, the diameter of the base of the cone widened and its top spread out, and at the same time the etch depth increased (Fig. 2(c)).

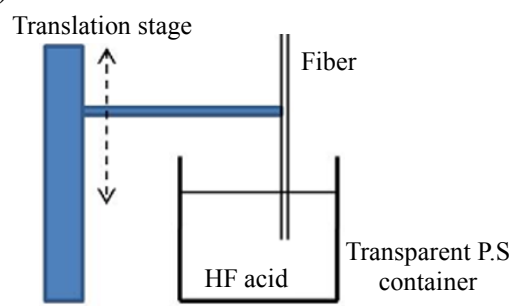

Fig. 1 Schematics of setup for chemical etching of fiber tips.

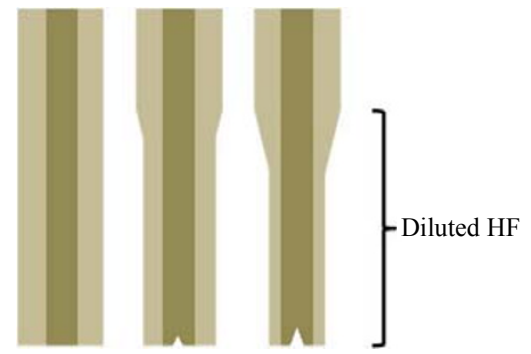

Fig. 2 Fiber tip formation during etching process.

For a fiber with a particular dopant the final tip shape can be designed by adjusting the HF concentration and the etching time. Microscope images of four etched SMFs and images of four multimode fibers have been illustrated in Figs. 3 and 4 respectively. We will later see how the geometry of fiber tip affects the output beam profile.

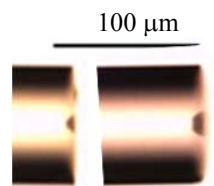

(a)

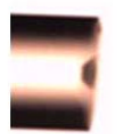

(c)

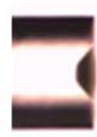

(d)
Fig. 3 Microscope image of the tip profile of three SMFs, after (a) $2 \mathrm{~min}$, (b) $4 \mathrm{~min}$, (c) $8 \mathrm{~min}$, and (d) $12 \mathrm{~min}$ etch in the same condition.

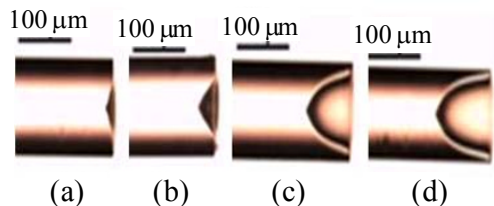

Fig. 4 Tip profile of four InfiniCor 300 multimode fibers (MMFs), after (a) $2 \mathrm{~min}$, (b) $3 \mathrm{~min}$, (c) $10 \mathrm{~min}$, and (d) $15 \mathrm{~min}$ etch in the same condition.

\section{Mode conversion in single mode fiber using concave tip fiber}

Figure 5 shows the schematic configuration of the core-etched and core/clad-etched single mode fibers, where $W_{\text {etch }}$ (etch width), $D_{\text {etch }}$ (etch depth) and $n_{\text {etch }}$ correspond to the base of cone, axis of cone, and refractive index of the etched region. We have assumed two cases: 1) when the etched region is confined to core only (Fig. 5(a)) and 2) where etched area is spread to cladding as well (Fig. 5(b)).

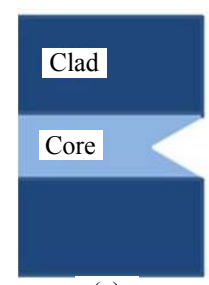

(a)

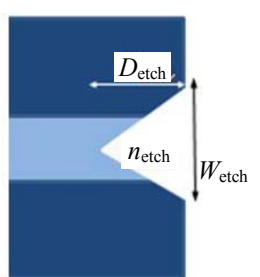

(b)
Fig. 5 Schematic of (a) core-etched single mode fiber and (b) core/clad-etched SMF.

We used the Rsoft commercial package for simulation, which implements beam propagation method to solve light propagation in the single mode fiber and free space as light exits the fiber [33]. Here we present the simulation results where the geometry of etched area has been optimized to form a flat top, donut or tight Gaussian shape beam, respectively on the tip of the fiber. In this simulation, the core and cladding diameters and refractive indices are $D_{\text {core }}=4 \mu \mathrm{m}, D_{\text {clad }}=125 \mu \mathrm{m}, n_{\text {core }}=1.4585$ and $\Delta n=0.005$ at wavelength of $633 \mathrm{~nm}$. Figure 6(a) shows the simulation result of output field of a regular single mode fiber as it propagates $10_{\mathrm{j}, \mathrm{m}} \mu \mathrm{m}$ in free space ( $z=0$ corresponds to tip of the fiber). As it is expected, the beam has a Gaussian distribution and maintains its profile as it propagates in free space. The depth, width and the refractive index of the etched region are the key parameters to determine the intensity profile of the output beam. Figures 6(b) and 6(c) represent the field propagation of an etched fiber with the depth of only $0.5 \mu \mathrm{m}$ at two distances of $z=3 \mu \mathrm{m}$ and $z=8 \mu \mathrm{m}$. A flat top profile with uniform intensity distribution starts to 
form at only $3 \mu \mathrm{m}$ away from the tip and widens as it propagates in free space and gradually an annular beam is formed as shown in Fig. 6(c), and then diverges. To obtain a beam profile with smaller spot size relative to the regular single mode fiber one can deep etch the tip and fill the etched volume with a higher index material. Figure 6(d) represents the beam propagation for a single mode fiber etched with the depth of $D_{\text {etch }}=3 \mu \mathrm{m}$ and then filled with a material of refractive index of 1.58. Light exiting from the etched tip is focused tightly.

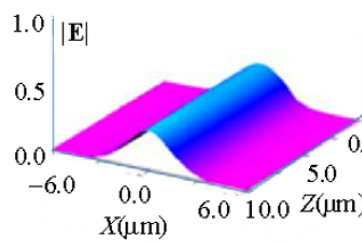

(a)

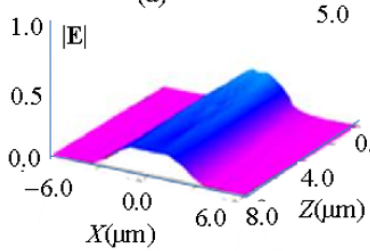

(c)

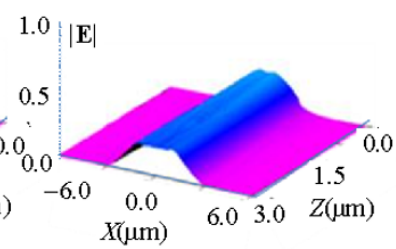

(b)

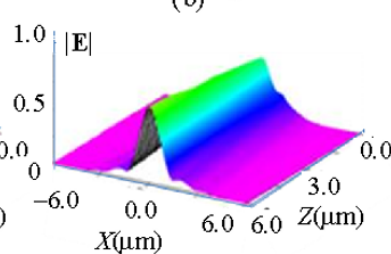

(d)
Fig. $63 \mathrm{D}$ representation of light propagation as it exits (a) an unetched single mode fiber (Gaussian profile), (b) and (c) etched depth $D_{\text {etch }}=0.5 \mu \mathrm{m}$ at $z=3 \mu \mathrm{m}$ and $z=8 \mu \mathrm{m}$ (flat profile), and (d) showing spot size reduction for $D_{\text {etch }}=3 \mu \mathrm{m}, W_{\text {etch }}=4 \mu \mathrm{m}$.

To characterize and compare the beam size reduction properties of an etched fiber with an un-etched SMF, we define several important parameters as follows. The optical mode field diameter (MFD) is defined as the full width at $1 / e$ of the maximum of the relative field intensity. The focus spot diameter is the smallest optical MFD along the light propagation distance, and the distance from the fiber tip to the minimum spot position is defined as the focus distance $\left(z_{f}\right)$.

The mode field diameters of the single mode optical fiber and of an integrated optical waveguide can both be approximated by a Gaussian field distribution [15]. For a guided step index fiber, the spot radius $W$ is half the $M F D$ and defined as the radial distance at which the field amplitude is $1 / e$ of its maximum. It is approximated by:

$$
W=M F D / 2=a\left(0.65+\frac{1619}{v^{\frac{3}{2}}}+\frac{2.879}{V^{6}}\right)
$$

where $a$ is the fiber core radius. The term $V$ is defined as

$$
V=\frac{2 \pi}{\lambda} a\left(n_{\text {core }}^{2}-n_{\text {clad }}^{2}\right)^{1 / 2}
$$

where $\lambda$ is the free-space wavelength, $n_{\text {core }}$ and $n_{\text {clad }}$ are the core and cladding refractive indices, and $\left(n_{\text {core }}^{2}-n_{\text {clad }}^{2}\right)^{1 / 2}$ is numerical aperture (NA) of the fiber. Equations (1) and (2) can be used to approximate the $M F D$ as a function of wavelength:

$$
M F D \alpha \frac{V \lambda}{\pi N A} .
$$

As demonstrated in (3), $M F D$ is directly proportional to wavelength. Therefore, neglecting dispersion in fiber, core diameter, $V$ number and $M F D$ are a multiple of wavelength. In our simulation model, we have selected a SMF with $n_{\text {core }}=1.46$ and $\Delta n=0.004, N A$ of 0.102 , and a core diameter of $6.3 \lambda$ which has to be etched and used for coupling into a high index waveguide. To study the focus spot parameter (MFD and position) of an etched fiber, several etch depths and widths (as multiple of wavelength) have been considered. Simulations are grouped based on etch depths wherein each group comprises of different widths except for $D_{\text {etch }}=0$ which corresponds to an unetched SMF. Note that, the control over etch depth and width is possible by adjusting the etching time and hydrofluoric (HF) dilution ratio from the experimental standpoint.

In the case of a concave tip SMF with etching depth of $8 \lambda$ and $n_{\text {etch }}=1.58$, the graph in Fig. 7 shows that the value of $M F D$ starts to fall to a minimum amount at focus spot $\left(z_{f}\right)$ marked by a solid line and rises afterward. Point $z=0$ corresponds to the apex in cone of etched region, while dashed line represents the tip of the fiber (base of cone of etch) thus positioned at $z=8 \lambda=D_{\text {etch. }}$. The minimum $M F D$ of $4 \lambda$ at the distance of $z_{f}=12.6 \lambda$ (Fig. 7) for etch width of $16 \lambda$ has been achieved. MFD then increases 
sharply and reaches to the value of $5.8 \lambda$ at $z=39.5 \lambda$. The width of etched region greatly influences the focus spot parameters such as focusing length and $M F D$. The minimum $M F D$ and focusing distance increases with etching widths. Note that, the spot size reduction begins inside the etched region (left of dashed line in Fig. 7).

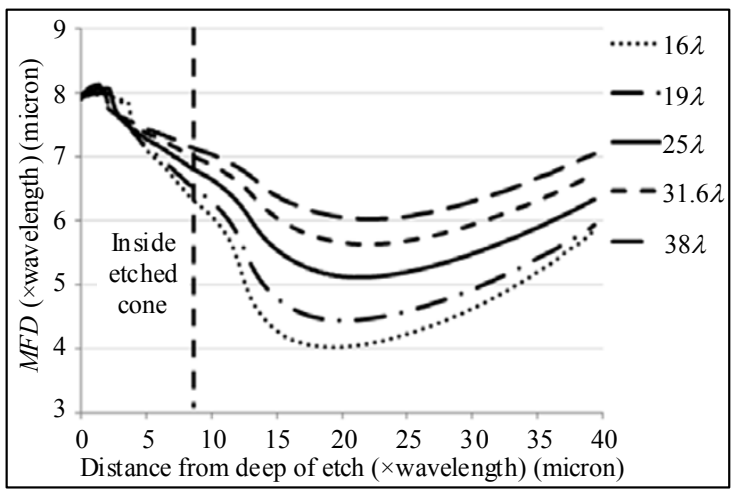

Fig. $7 M F D$ as a function of distance from depth of etched cone for $D_{\text {etch }}=8 \lambda$ and different $W_{\text {etch }}$, refractive index $n_{\text {etch }}=1.58$ (solid line represents the minimum spot size location and dashed line corresponds to the location of fiber tip).

In butt-coupling of fiber to integrated optical waveguides in which the fiber tip is in contact with the waveguide face, having a fiber with a flat end face is desirable. So concave tip fiber in contrast with the lensed fibers or fibers with diffractive elements on the tip, is more reliable in contact with the waveguide thus providing easy handling and simple packaging. A concave tip fiber is easy to align if the focusing spot (minimum spot size) forms exactly on the tip of the fiber in contrast to the other modified-tip fibers, which alignment plays an important role in order to position the waveguide input in the focal point of the lensed fibers.

As seen in Fig. 7 for an etch depth of $8 \lambda$ the focal point forms at some distances away from the fiber tip. Further simulations show that at the etching depth of around $25 \lambda$, the minimum spot size location falls exactly on the fiber tip for several etching widths (dashed line and the solid line overlap). Graphs in Fig. 8 demonstrate the MFD reduction property of five concave tip fibers with
$D_{\text {etch }}=25 \lambda$ with five different etched widths, $W_{\text {etch }}$. The minimum $M F D$ is achieved for $W_{\text {etch }}$ of $50 \lambda$. Likewise our simulation reveals that for an etch depth of $28 \lambda$ the minimum spot size $(M F D=4 \lambda)$ is half the original beam size $(M F D=8 \lambda)$ for $W_{\text {etch }}$ of $56 \lambda$. However, the focusing distance is less than etch depth $\left(z_{f}<D_{\text {etch }}\right)$ and the minimum spot size forms inside the fiber. Subsequently, light starts to diverge and as a result, $M F D$ increases.

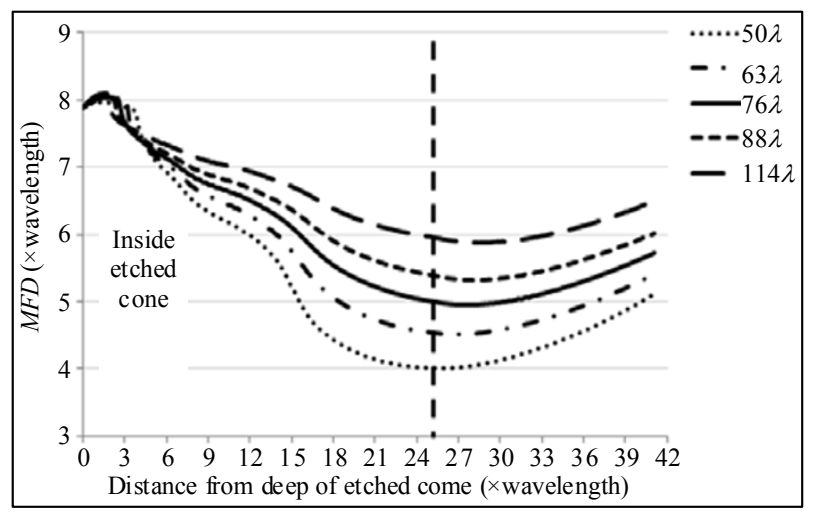

Fig. $8 M F D$ as a function of distance from depth of etched cone for $D_{\text {etch }}=25 \lambda$ and different $W_{\text {etch, refractive index }}$ $n_{\text {etch }}=1.58$ (solid line represents the minimum spot size location and dashed line corresponds to the location of fiber tip).

A careful study of the etching parameters reveals that the minimum $M F D$ of $4 \lambda$ is attainable for all etching depths, by modifying the etch width. It has been realized that the ratio between the half etch width and etch depth $\left(W_{\text {etch }} / 2 D_{\text {etch }}\right)$ has remained constant in minimum spot size condition, which implies that for each wavelength there is corresponding lens that provides minimum spot size.

\section{Experimental observation of mode size reduction in single mode fiber}

The fabrication method and characterizations of etched fibers were described in Section 2. In this section, experimental observation of mode conversion for several concave tip fibers will be explained. For this experiment, we used conventional Ge-doped step-index SMFs with $n_{\text {core }}=1.46$ and $\Delta n=0.004, N A$ of 0.102 , clad diameter of $125 \mu \mathrm{m}$, and a core diameter of $4 \mu \mathrm{m}$ at 
wavelength $633 \mathrm{~nm}$. Figure 3 shows the microscope images of a few etched fibers. The output profile of each concave tip fiber has been examined using the experimental setup shown in Fig. 9. The laser used for this study was a He-Ne laser providing up to $20 \mathrm{~mW}$ power. A polarizer was inserted after laser to provide control over injected power. The intensity profile of fibers was imaged onto a charge coupled device (CCD) camera using a $20 \times$ lens and measured by commercial laser beam profiler software. The images obtained with the CCD camera for un-etched SMF and etched fibers with various etching depths and widths are shown in Fig. 10. Our experimental measurement matched theoretical predictions very well as depicted in Fig. 11. In this figure a comparison is made between the results from simulation (solid line) and experiment (data points) for five particular fibers for given parameters at wavelength $633 \mathrm{~nm}$.

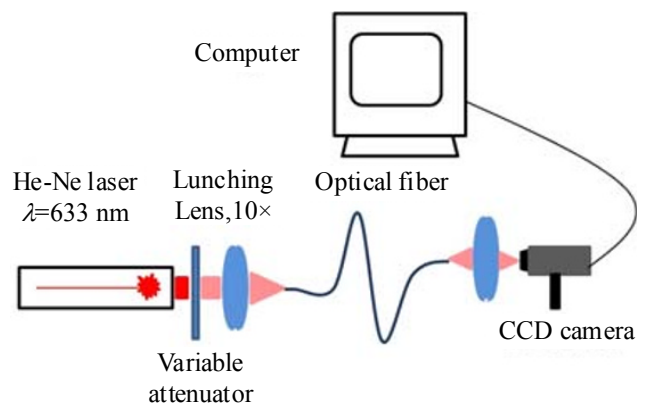

Fig. 9 Schematic of the experimental setup.

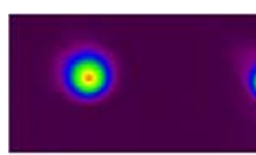

(a) ○ $\circ$

(b)

(c)

(d)

Fig. 10 Intensity distribution profile of (a) unetched SMF, (b) $W_{\text {etch }} \times D_{\text {etch }}=16 \times 5 \mu \mathrm{m}^{2}$, and (c) $28 \times 11 \mu \mathrm{m}^{2}$, and (d) $80 \times 18 \mu \mathrm{m}^{2}$ imaged at their corresponding focal length $\left(n_{\text {etch }}=1.58\right)$.

Concave tip fiber behaves like a fiber with micro-lens at the tip, but unlike spherical micro-lens, the fiber end face remains flat therefore contact alignment with an integrated optical waveguide would be possible. Here we give an analysis for the butt-coupling from concave tip fibers and two high index contrast integrated optical waveguides (e.g. silicon-on-insulator (SOI) and silicon oxynitride ( $\mathrm{SiON}$ ) sandwiched between $\mathrm{SiO}_{2}$ layers), the end of which is in contact with the tip of the fiber.

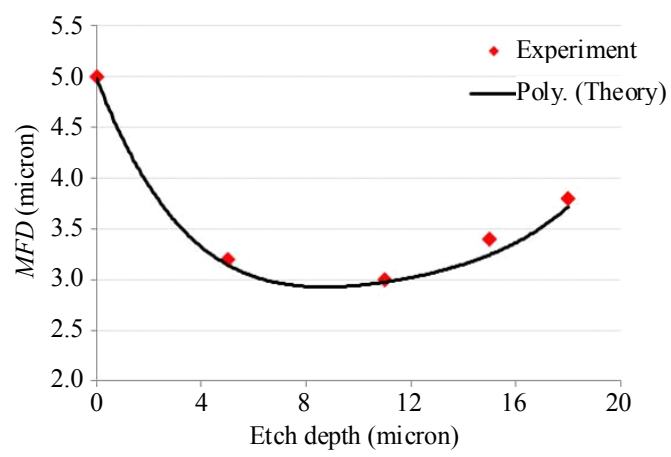

Fig. 11 Simulated and measured $M F D$ for different etch depths.

First, we present a waveguide structure based on SOI. A single mode Si waveguide with the width of $w_{\mathrm{wg}}=450 \mathrm{~nm}$ and refractive index of $n_{\mathrm{si}}=3.5$ and cladding index of $n_{\mathrm{Sio}_{2}}=1.444$ for a wavelength of $1550 \mathrm{~nm}$ has been considered. The top Si layer of the SOI wafer is $250 \mathrm{~nm}$ thick. In our simulation model, we have used a SMF with $n_{\text {core }}=1.444, \Delta n=0.007$, $N A$ of 0.143 , and a core diameter of $5.3 \lambda$ which has to be etched and used for coupling into a high index waveguide. Simulations show that for etching depth of around $17 \lambda$, the minimum spot size location falls exactly on the fiber tip for several etching widths. The minimum $M F D$ of $3.1 \lambda$ equivalent of $4.8 \mu \mathrm{m}$ at wavelength $1550 \mathrm{~nm}$ is achieved corresponding to $W_{\text {etch }}$ of $26 \lambda$.

The coupling loss is defined as the transition loss from the field distribution at the junction to the fundamental mode of the single mode SOI waveguide. A typical single mode fiber at $1550 \mathrm{~nm}$ wavelength region has the $M F D$ of around $9 \mu \mathrm{m}$. When simply butt-coupling the fiber and nanometers size SOI waveguide, the coupling efficiency from the fiber to the waveguide is only $0.8 \%$, indicating a loss of $20.6 \mathrm{~dB}$. However, the concave tip fiber method can achieve a much closer match between the $M F D$ of waveguide and fiber thus improving the coupling efficiency.

As mentioned above, it is possible to obtain 
minimum spot size on the fiber's tip, which makes it suitable for butt-coupling application. Plot in Fig. 12 represents the coupling loss vs. $M F D$ while coupling into the SOI waveguide with above dimensions, width $w_{\mathrm{wg}}=450 \mathrm{~nm}$ and refractive index of $n_{\mathrm{Si}}=3.5$, cladding index of $n_{\mathrm{Si}_{2}}=1.444$. In this figure, the dashed-dot curves represent the coupling loss of fiber to SOI waveguide. For $M F D$ s smaller than $9 \mu \mathrm{m}$, the coupling loss is decreased due to the reduced modal mismatch. Coupling loss has been reduced from $21 \mathrm{~dB}$ for an unetched fiber (equivalent of $M F D=9 \mu \mathrm{m})$ to $15 \mathrm{~dB}$ in concave tip fiber with a reduced $M F D$ of $4.8 \mu \mathrm{m}$. In order to further improve the coupling efficiency between a nano-size SOI waveguide and a concave tip SMF, a lateral linear adiabatic taper [34] should be integrated with the waveguide to gradually transform a large mode size of fiber into a small mode size of SOI waveguide. Combining concave tip fiber method with laterally tapered waveguide scheme improves the coupling efficiency from $1 \%$ to $48 \%$ or the loss will drop from $21 \mathrm{~dB}$ to $4 \mathrm{~dB}$. This is a significant improvement and will have major impact on the implementation of integrated optical devices in the areas of sensing and communication.

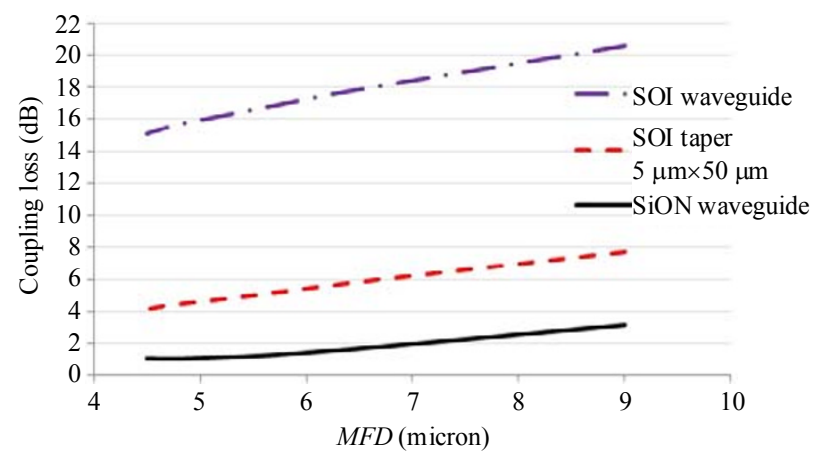

Fig. 12 Simulated and measured $M F D$ for different etch depths.

We have also observed major improvement in coupling light from fiber to $\mathrm{SiON}$ waveguides. $\mathrm{SiON}$ is a promising candidate for photonic integrated circuits, as it can be conveniently deposited with low-pressure chemical vapor deposition or plasma enhanced chemical vapor deposition and has good optical properties around $1550 \mathrm{~nm}$. Its refractive index can vary from 1.45 to 2 . Thus, for a SiON waveguide enclosed in silicon dioxide, both the width and thickness of the waveguide can be micron scale for single mode propagation. We have considered a similar approach for coupling light from a single mode fiber to $\mathrm{SiON}$ waveguide. A SiON waveguide with $w_{\mathrm{wg}}=2.5 \mu \mathrm{m}$, refractive index of $n_{\text {core }}=1.49$, and cladding index of $n_{\mathrm{Sio}_{2}}=1.445$ at a wavelength of $1550 \mathrm{~nm}$ has been considered here. Our results show the coupling loss will be reduced from $3.5 \mathrm{~dB}$ to $1 \mathrm{~dB}$, which is equivalent to improving the coupling efficiency from $45 \%$ to $80 \%$.

\section{Experimental observation of beam shaping using concave tip single mode fiber}

In Section 3, we theoretically demonstrated that concave tip SMFs with submicron etch depth can transform a Gaussian shaped beam into a flat top with a uniform intensity distribution while a micron size etch concave tip fiber creates a donut profile with a low intensity in the center. Here, we present the experimental result demonstrating the output behavior of two concave tip SMFs etched with different etched depths.

Two pieces of Ge-doped core SMFs (at $633 \mathrm{~nm}$ ) have been selected for this experiment and a similar procedure as described in Section 2 was carried out for etching the fiber. These fibers were in $49 \% \mathrm{HF}$, diluted with water for $3 \mathrm{~min}$ and 4 min respectively. The output profile of each fiber was examined and compared with an unetched SMF using the setup shown in Fig. 9. In order to image the output profile of the fiber tip into a far-field, the position of fiber end-face was at the focal plane of a $40 \times$ objective lens.

As shown in Fig. 13(a), the intensity distribution of a step index Ge-doped core SMFs is Gaussian while the output beam profile of fiber that is etched for 3 min produces a flat top beam with uniform 
intensity (Fig. 13(b)). In order to estimate the flatness of a beam we have chosen full width-half maximum (FWHM) method. The flatness is defined as the ratio of flat diameter (measured from $96 \%$ to $100 \%$ of the relative power level) to spot diameter (measured at $50 \%$ of the relative power level) [10-12]. A flat top beam with intensity variation of approximately $5 \%$ and $50 \%$ of flat top diameter to spot diameter ratio has been achieved. Intensity distribution plot in Fig. 13(c) corresponds to the output beam profile of the fiber that is etched for 4 min. In this case an annular beam profile has been generated.

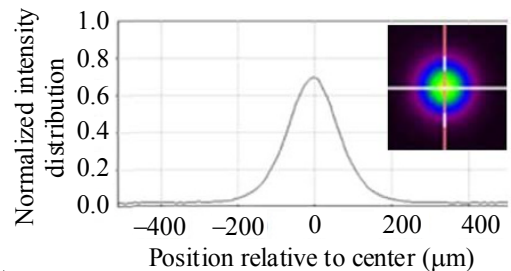

(a)

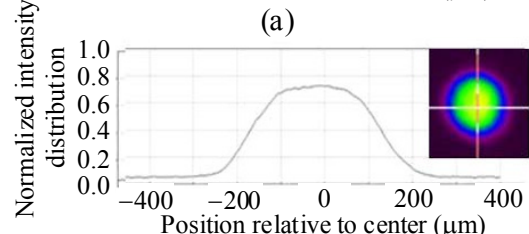

(b)

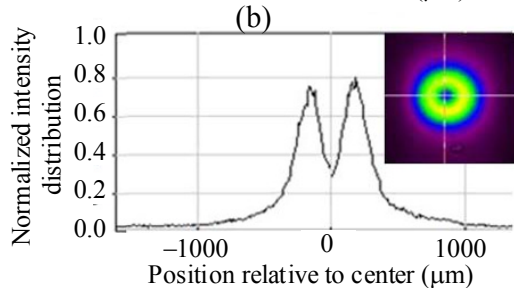

(c)

Fig. 13 Image of intensity distribution profile (left) and a plot corresponding to a vertical slice (right) of (a) unetched SMF, (b) fiber etched for $3 \mathrm{~min}$, and (c) fiber etched for $4 \mathrm{~min}$.

\section{Beam shaping using concave tip multimode fiber}

For a standard MMF such as InfiniCor 300 with germanium oxide $\left(\mathrm{GeO}_{2}\right)$ doped core and silica cladding, the etching process is similar to process described in Section 2. In this type of fibers a large number of modes exist where ratios of their amplitudes and phases relative to each other randomly vary due to environmental fluctuations. In our simulations we used random mode generator to ensure our results represent the most general case. We then examined the output profile of an etched fiber using the setup demonstrated in Fig. 9. The fiber modes were dynamically scrambled to obtain a time average of intensity distribution [36].

The output beam profile of an etched multimode fiber is shown in Fig. 14. Intensity distribution plot in Fig. 14(a) corresponds to an etched MMF at distance $0.5 \mathrm{~mm}$ away from the fiber. Figure 14(b) presents the intensity distribution at the distance of $2 \mathrm{~mm}$ away from the fiber tip. A flat top beam with intensity variation of approximately $5 \%$ and $67 \%$ of flat top diameter to spot diameter ratio has been achieved as predicted by our simulations. The results in Fig. 14(b) demonstrate a spot size diameter of about $0.5 \mathrm{~mm}$ at a distance of $2 \mathrm{~mm}$ away from the fiber tip. The evolution of intensity profile after light exiting the fiber is as follow: 1) the beam initially makes a transition from Gaussian into a flat top at the propagation distance of $2 \mathrm{~mm}$ from fiber as illustrated in Fig. 14(b) and 2) for a very short distance of a few microns relative to flat top plane, the beam essentially retains its shape, with only a slight degradation of uniformity. But at larger distances (e.g. $2.1 \mathrm{~mm}$ and $2.5 \mathrm{~mm}$ from the fiber tip), beam starts to diverge and a low-intensity hole develops on the beam axis. The intensity profile at the distances of $2.1 \mathrm{~mm}$ and $2.5 \mathrm{~mm}$ from the fiber tip is shown in Figs. 14(c) and 14(d). This figure clearly illustrates that as the beam expands, the intensity at the center of beam reduces and an annular intensity profile is generated.

Donut shape intensity distribution is particularly useful for medical application for treatment of the interior of hollow body organs [37-39]. There are several methods to deliver a donut beam directly from the fiber to the surface such as integrated optical fiber with scattering tips or micro-lenses [37-39], all-fiber LPG [10] and multimode interference devices [40]. The LPG and multimode interference based techniques do not meet the high 
power delivery requirements. Compared to currently-used modified tips [37-39], concave tip fibers have several advantages such as precise control of beam size, high transmission efficiency, uniform intensity distribution, and low cost of fabrication process.

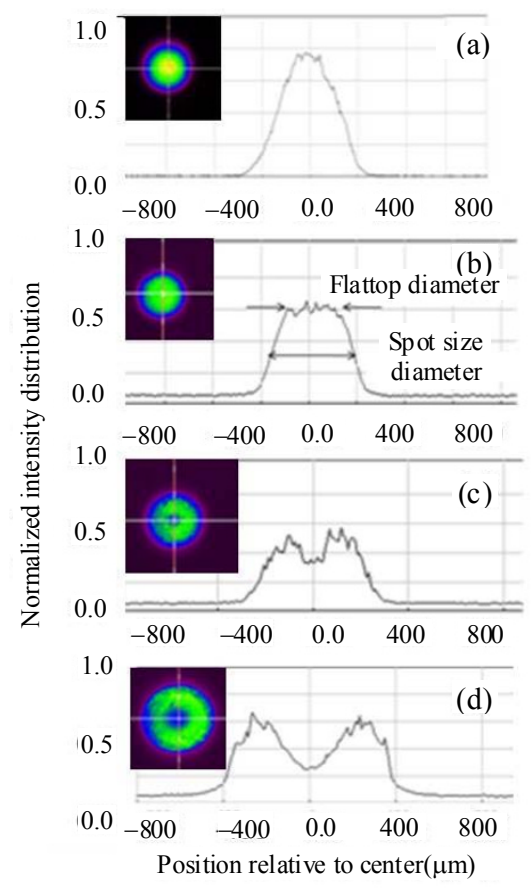

Fig. 14 Image of intensity profile (left) and its corresponding plot associated with a vertical slice (right): fiber tip is about (a) $0.5 \mathrm{~mm}$, (b) $2 \mathrm{~mm}$, (c) $2.1 \mathrm{~mm}$, and (d) $2.5 \mathrm{~mm}$ away from CCD camera.

In some practical applications, a flat beam might be needed at a given distance from the fiber end face. In order to maintain the flat beam profile in a desired distance from end face, as a test case we used a $20 \times$ microscope objective lens to provide a near-field image of a flat top output from an etched multimode fiber at a given distance from the lens. In a test experiment we tried to generate a flat-top beam at 3-cm distance from an imaging lens inserted between the fiber end and camera in experimental setup (Fig. 9). Figure 15 represents a flat beam profile with a 1-mm diameter which has been imaged on the CCD $3 \mathrm{~cm}$ away from the objective lens. A flat top beam with intensity variation of approximately $5 \%$ and $84 \%$ of flat top diameter to spot diameter ratio has been achieved. In comparison with the direct image of the beam (Fig. 14(b)), this ratio has been improved significantly, from $67 \%$ to $84 \%$.

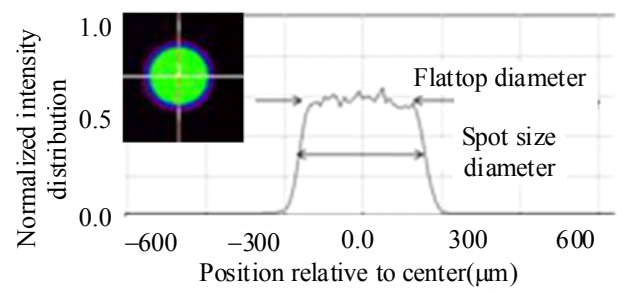

Fig. 15 Image of intensity profile (left) and the corresponding vertical slice (right) of a flat top beam imaged by a $20 \times$ microscope objective lens at a distance of $3 \mathrm{~cm}$ away from the lens $(\lambda=633 \mathrm{~nm})$.

Previously, the highest reported flat top to spot diameter ratio was $58 \%$ using a single mode fiber abrupt taper [12] and 50\% in LPG [10, 11], suggesting that the concave tip fibers have higher efficiency in delivering a uniform intensity, not to mention that LPG based devices are not applicable to high power delivery fiber system.

Our design of beam shaping optics departs from other reported works in several important aspects. Unlike refractive and diffractive optical elements, our device shows high efficiency, high fabrication tolerance, and easy alignment control. Proposed device is wavelength insensitive and has very low fabrication cost. In contrast to other all-fiber laser beam shaping devices, concave tip MMFs deliver a highly uniform beam directly from MMF which is desired for high power laser beam shaping applications. Also wavelength insensitive characteristic of this device make it distinguished from other similar fiber delivery designs.

Different applications may demand different multimode fibers, but this beam shaping technique is applicable to any multimode fiber. Assuming that the scattering associated with the roughness of fiber tip is negligible, there is no other source of loss in concave etched beam shaping device. Because beam uniformity and efficient power delivery are desirable in the field of medicine and industrial laser marking, 
proposed device can substitute the complex bulky optical elements in these applications. By tuning the etching time, we have shown that an appropriate fiber tip could be made to control the beam profile from flat to donut which is often required for some medical applications [37-39]. We have applied this beam flattening technique for nerve stimulation [41]. The cavernous nerves $(\mathrm{CN})$ along the prostate surface are responsible for erectile function. Improved identification and preservation of the CN's is critical to maintaining sexual potency after prostate cancer surgery. Optimal design of the fiber optic delivery system is critical for safe, reproducible stimulation during the surgery. In a direct comparison of the magnitude and response time of the intracavernosal pressure (ICP) for both Gaussian and flat-top spatial beam profiles it was found that the flat-top laser beam profile consistently produced a faster and higher ICP response at a lower radiant exposure than the Gaussian beam profile [41].

\section{Summary}

We have introduced a novel all-fiber beam shaping device by inverse etching the tip of a multimode fiber. Details of this realization, together with a fabrication method and experimental results have been presented. We have been able to achieve a flat top beam with intensity variation of approximately $5 \%$ and $67 \%$ of flat top diameter to spot diameter ratio in multimode fibers. The flat to spot diameter ratio was improved to $84 \%$ by deploying a lens to generate a flat beam at a targeted distance. Unlike the other proposed beam shaping techniques, concave tip fibers have no loss (neglecting the scattering on the fiber tip). The operational performance of this beam shaping device is independent of wavelength, which makes it compatible with all kinds of lasers in different wavelength regions. This technique is attractive for applications which require high power, uniform intensity distribution delivered directly from fibers.
In comparison with other beam flattening methods, this method offers a considerably simpler and cheaper fabrication process.

We have also introduced a new mode conversion mechanism for single mode fiber by inverse etching of the fiber tip and incorporation of high index material in the etched region. With this technique light could be focused on the fiber tip allowing high efficiency coupling of light from an optical fiber to an integrated optical waveguide.

Open Access This article is distributed under the terms of the Creative Commons Attribution License which permits any use, distribution, and reproduction in any medium, provided the original author(s) and source are credited.

\section{References}

[1] B. R. Frieden, "Lossless conversion of a plane wave to a plane wave of uniform irradiance," Appl. Opt., vol. 4, no. 11, pp. 1400-1403, 1965.

[2] F. M. Dickey, S. C. Holswade, and D. L. Shealy, Laser beam shaping applications, Taylor Francis Group, Boca Raton, USA: CRC Press, 2006.

[3] S. P. Chang, J. M. Kuo, Y. P. Lee, C. M. Lu, and K. J. Ling, "Transformation of Gaussian to coherent uniform beams by inverse-Gaussian transmittive filters," Appl. Opt., vol. 37, no. 4, pp. 747-752, 1998.

[4] C. C. Aleksoff, K. K. Ellis, and B. D. Neagle, "Holographic conversion of a Gaussian beam to a near-field uniform beam," Opt. Eng., vol. 30, no. 5, pp. 537-543, 1991.

[5] M. Quintanilla and A. M. de Frutos, "Holographic filter that transforms a Gaussian into a uniform beam," Appl. Opt., vol. 20, no. 5, pp. 879-880, 1981.

[6] C. Y. Han, Y. Ishii, and K. Murata, "Reshaping collimated laser beams with Gaussian profile to uniform profiles," Appl. Opt., vol. 22, no. 22, pp. 3644-3647, 1983.

[7] J. A. Hoffnagle and C. M. Jefferson, "Design and performance of a refractive optical system that converts a Gaussian to a flattop beam," Appl. Opt., vol. 39, no. 30, pp. 5488-5499, 2000.

[8] P. W. Rhodes and D. L. Shealy, "Refractive optical systems for irradiance redistribution of collimated 
radiation: their design and analysis," Appl. Opt., vol. 19, no. 20, pp. 3545-3553, 1980.

[9] J. R. Hayes, J. C. Flanagan, T. M. Monro, and D. J. Richardson, "Square core jacketed air-clad fiber," Opt. Exp., vol. 14, no. 22, pp. 10345-10350, 2006.

[10] X. Gu, W. Mohammed, L. Qian, and P. W. E. Smith, "All-fiber laser beam shaping using a long-period grating," IEEE Photonics Technology Letters, vol. 20, no. 13, pp. 1130-1132, 2008.

[11] W. Mohammed and X. Gu, "Long-period grating and its application in laser beam shaping in the $1.0 \mu \mathrm{m}$ wavelength region," Appl. Opt., vol. 48, no. 12, pp. 2249-2254, 2009.

[12] M. Sumetsky and S. Ramachandran, "Multiple mode conversion and beam shaping with superimposed long period gratings," Opt. Exp., vol. 16, no. 1, pp. 402-412, 2008.

[13] Zh. Tian, M. Nix, and S. S-H. Yam, "Laser beam shaping using a single-mode fiber abrupt taper," Opt. Lett., vol. 34, no. 3, pp. 229-231, 2009.

[14] M. Prasciolu, D. Cojoc, S. Cabrini, L. Businaro, P. Candeloro, M. Tormen , R. Kumar, C. Liberale, V. Degiorgio, A. Gerardino, G. Gigli, D. Pisignano, E. Di Fabrizio, and R. Cingolani, "Design and fabrication of on-fiber diffractive elements for fiber-waveguide coupling by means of e-beam lithography," Microelectronic Engineering, vol. 67-68, no. 1, pp. 169-174, 2003.

[15] F. Schiappelli, R. Kumar, M. Prasciolu, D. Cojoc, S. Cabrini, M. De Vittorio, G. Visimberg, A. Gerardino, V. Degiorgio, and E. Di Fabrizio, "Efficient fiber-to-waveguide coupling by a lens on the end of the optical fiber fabricated by focused ion beam milling," Microelectronic Engineering, vol. 73-74, pp. 397-404, 2004.

[16] D. Taillaert, W. Bogaerts, P. Bienstman, T. F. Krauss, P. V. Daele, I. Moerman, S. Verstuyft, K. D. Mesel, and R. Baets, "An out-of-plane grating coupler for efficient butt-coupling between compact planar waveguides and single-mode fibers," IEEE J. Quantum Electron., vol. 38, no. 7, pp. 949-955, 2002.

[17] B. Wang, J. Jiang, and G. Nordin, "Compact slanted grating couplers," Opt. Express, vol. 12, no. 15, pp. 3313-3326, 2004.

[18] G. Masanovic, G. Reed, W Headley, B. Timotijevic, V. Passaro, R. Atta, G. Ensell, and A. Evans, "A high efficiency input/output coupler for small silicon photonic devices," Opt. Exp., vol. 13, no. 19, pp. 7374-7379, 2005.

[19] Z. Lu, "Efficient fiber-to-waveguide coupling through the vertical leakage from a microring," Opt. Lett., vol. 32, no. 19, pp. 2861-2863, 2007.

[20] T. Dillon, J. Murakowski, S. Shi, and D. Prather, "Fiber-to-waveguide coupler based on the parabolic reflector," Opt. Lett., vol. 33, no. 9, pp. 896-898, 2008.

[21] A. Sure, T. Dillon, J. Murakowski, C. Lin, D. Pustai, and D. Prather, "Fabrication and characterization of three-dimensional silicon tapers," Opt. Exp., vol. 11, no. 26, pp. 3555-3561, 2003.

[22] B. Luyssaert, P. Vandersteegen, D. Taillaert, P. Dumon, W. Bogaerts, P. Bienstman, D. V. Thourhout, V. Wiaux, S. Beckx, and R. Baets, "A compact photonic horizontal spot-size converter realized in silicon-oninsulator," IEEE Photon. Technol. Lett., vol. 17, no. 1, pp. 73-75, 2005.

[23] J. H. Schmid, B. Lamontagne, P. Cheben, A. Delâge, S. Janz, A. Densmore, J. Lapointe, E. Post, P. Waldron, and D. X. Xu, "Mode converters for coupling to high aspect ratio Silicon-on-insulator channel waveguides," IEEE Photon. Technol. Lett., vol. 19, no. 11, pp. 855-857, 2007.

[24] G. Roelkens, P. Dumon, W. Bogaerts, D. Van Thourhout, and R. Baets, "Efficient silicon-on-insulator fiber coupler fabricated using 248-nm-deep UV lithography," IEEE Photon. Technol. Lett., vol. 17, no. 12, pp. 2613-1615, 2005.

[25] V. R. Almeida, R. R. Panepucci, and M. Lipson, "Nano-taper for compact mode conversion," Opt. Lett., vol. 28, no. 15, pp. 1302-1315, 2003.

[26] D. B. Talley, L. B. Shaw, J. S. Sanghera, I. D. Aggarwal, A. Cricenti, R. Generosi, M. Luce, G. Margaritondo, J. M. Gilligan, and N. H. Tolk, "Scanning near field infrared microscopy using chalcogenide fiber tips," Mater. Lett., vol. 42, no. 5, pp. 339-344, 2000.

[27] E. Betzig and R. J. Chichester, "Single molecules observed by near-field scanning optical microscopy," Science, vol. 262, no. 5138, pp. 1422-1425, 1993.

[28] L. Thiery, N. Marini, J. P. Prenel, and M. Spajer, C. Bainier, and D. Courgon, "Temperature profile measurements of near-field optical microscopy fiber tips by means of sub-micronic thermocouple," Int. J. Therm. Sci., vol. 39, no. 4, pp. 519-525, 2000.

[29] B. A. F. Puygranier and P. Dawson, "Chemical etching of optical fiber tips- experiment and model," Ultramicroscopy, vol. 85, no. 4, pp. 235-248, 2000.

[30] D. J. Monk, D. S. Soane, and R. T. Howe, "A review of the chemical reaction mechanism and kinetics for hydrofluoric acid etching of silicon dioxide for surface micromachining applications," Thin Solid Films, vol. 232, no. 1, pp. 1-12, 1993. 
[31] N. E. Demagh, A. Guessoum, and H. Aissat, "Chemical etching of concave cone fiber ends for core fiber alignment," Meas. Sci. Technol., vol. 17, no. 1, pp. 119-122, 2006.

[32] S. T. Tso and J. A. Pask, "Reaction of glasses with hydrofluoric acid solution," J. Am. Ceram. Soc., vol. 65, no. 7, pp. 360-362, 1982.

[33] RSoft design group, Beam Prop user manual (version 8.1), Ossining, NY, USA.

[34] I. Moerman, P. P. Van Daele, and P. M. Demeester. "A review on fabrication technologies for the monolithic integration of tapers with III-V semiconductor devices," IEEE J. Select. Topics Quantum Electron., vol. 3, no. 6, pp. 1308-1320, 1997.

[35] J. Ch. Tuck, R. Hague, and C. Doyle, "Low cost optical fiber based Fabry-Perot strain sensor production," Meas. Sci. Technol., vol. 17, no. 8, pp. 2206-2212, 2006.

[36] M. W. Greenaway, W. G. Proud, and J. E. Field, "The development and study of a fiber delivery system for beam shaping," Rev. Sci. Instrum., vol. 73, no. 5, pp. 2185-2189, 2002.
[37] V. Russo, G. Righini, S. Sottini, and S. Trigari, "Axially- and side- radiating optical fibers for medical applications," Proc. SPIE, vol. 492, pp. 466-473, 1985.

[38] V. Russo, "Optical fiber delivery systems for laser angioplasty and laser treatment of tumours," Lasers in medical science, vol. 3, no. 1-4, pp. 207-211, 1988.

[39] F. Fankhauser, P. Rol, and S. Kwasniewska, "Integrated optical fiber systems - some theoretical aspects," International Ophthalmology, vol. 13, no. 4, pp. 239-242, 1989.

[40] Yig. O. Yilmaz, A. Mehta, W. S. Mohammed, and E. G. Johnson, "Fiber-optic beam shaper based on multimode interference," Opt. Lett., vol. 32, no. 21, pp. 3170-3172, 2007.

[41]S. Tozburun, G. A. Lagoda, M. Mayeh, A. L. Burnett, F. Farahi, and N. M. Fried, "A compact laparoscopic probe for optical stimulation of the prostate nerves," IEEE Journal of Selected Topics in Quantum Electronics, vol. 16, no. 4, pp. 941-945, 2010. 